\title{
Automatic Detection of Electrocardiogram ST Segment: Application in Ischemic Disease Diagnosis
}

\author{
Duck Hee Lee ${ }^{1}$, Jun Woo Park ${ }^{2}$, Jeasoon Choi ${ }^{3}$, Ahmed Rabbi ${ }^{1}$ and Reza Fazel-Rezai ${ }^{1}$ \\ ${ }^{1}$ Department of Electrical Engineering, University of North Dakota, Grand Forks, North Dakota, USA \\ ${ }^{2}$ Korea Artificial Organ Center, College of Medicine, Korea University, Seoul, South Korea \\ ${ }^{3}$ Medical Engineering R\&D Center, Asan Institute for Life Science, Asan Medical Center and \\ University of Ulsan College of Medicine, Seoul, South Korea
}

\begin{abstract}
The analysis of electrocardiograph (ECG) signal provides important clinical information for heart disease diagnosis. The ECG signal consists of the $P, Q R S$ complex, and $T$-wave. These waves correspond to the fields induced by specific electric phenomenon on the cardiac surface. Among them, the detection of ischemia can be achieved by analysis the ST segment. Ischemia is one of the most serious and prevalent heart diseases. In this paper, the European database was used for evaluation of automatic detection of the ST segment. The method comprises several steps; ECG signal loading from database, signal preprocessing, detection of QRS complex and R-peak, ST segment, and other relation parameter measurement. The developed application displays the results of the analysis.
\end{abstract}

Keywords-Electrocardiogram (ECG); Ischemia; European STT database; QRS complex; ST segment.

\section{INTRODUCTION}

Electrocardiographic (ECG) signals information is derived from analysis of the information indirectly reflected on the surface ECG. The ECG signal is able to make of basic information for heart disease, indisposed of the autonomic nervous system and stress. The world Health Organization estimates that 17.5 million people died of cardiovascular disease. It is representing $30 \%$ of all global deaths. Out of these, 7.6 million were due to coronary artery disease (CAD)[1]. During the last few years, a lot of research has provided the solution of analysis and diagnosis in ECG by adopting new technologies and algorithms. Among them, ischemic heart disease constitutes one of the most common fatal diseases in the world. Myocardial ischemia is caused by a lack of sufficient blood flow to the contractile cells and many lead to myocardial information with its severe sequel of heart failure, arrhythmias and death [2]. The ischemic disease is usually identified in the standard ECG by changes in values of measured amplitudes, times and durations on the ST-T complex. The ST-T complex of the ECG reflects the time period from the end of active ventricular depolarization to the end of depolarization in the heart cycle [3-4]. Therefore, STsegment changes are common ECG signal markers of important Myocardial ischemia [5]. The several methods for ischemia parameter detection ( $\mathrm{T}$ wave and ST complex) have been proposed. In generally, all of them are based on the spectral estimation [6] and signal point from the ST segment better characterizes ischemic patterns [3, 7]. The various methods have been applied to the ECG for ischemia analysis and detection: used the First Fourier Transform (FFT) to analyze the frequency component [8], fuzzy-logic, neural network, genetic algorithm, support vector machines (SVM), wavelet transform and many more [9]. However, most of the algorithms have sensitivity above the $80 \%$. In this study we consider two applications of the ST segment detection and display program: Detection of ischemia episodes and monitoring PC programming. The modifications in the shape parameters have been used for ST segment measurement.

\section{Method AND MATERIAL}

\section{A. European ST-T Database}

The European ST-T database [10-11] is intended to be used for evaluation of algorithm for analysis of ST and $\mathrm{T}$ wave changes. It consists of three following files: 1) Header file has the patient's information, lead, medication, clinical findings and recording equipment information. 2) Data file has an ECG data recording format that is of double-channel 2-hour length, resolution of 12 bits and $250 \mathrm{~Hz}$ sampling frequency, and data format of MIT-212 format. 3) Finally, annotation file contains data information (data beat, ST and T change start-peak-end, noise, rhythm). It includes numerous ischemic episodes of all types and thus it is very useful in evaluating ischemic detection algorithms.

\section{B. Description of the detection system}

This system is intelligent computer applications that provide decision support through acquisition and processing of human experts knowledge. In order to operate the plotting signal and detection algorithms through European ST-T database signal, we used a $2.5 \mathrm{GHz}$ Pentium processor, $2 \mathrm{Gbyte}$ of RAM and the Microsoft ${ }^{\mathrm{TM}}$ Visual $\mathrm{C}++6.0$ programming tools. Figure 1 show the block diagram of the automatic detection of ST change and segment system. This system comprises three separate parts: an ECG data loading and open from the database, signal parameter detection of ECG signal, and ST measurement of the ischemia disease.

\section{Signal Preprocessing}

The infinitesimal ECG signal have included for various noise. Accordingly, noise has to be reduced before the signal processing. The source of noise is encountered at every stage of data acquisition until the data is digitized. Power noise, muscular contract noise, electrode movement with signal wandering, and analog-to-digital converter noise all perturb the ECG signals [12]. 


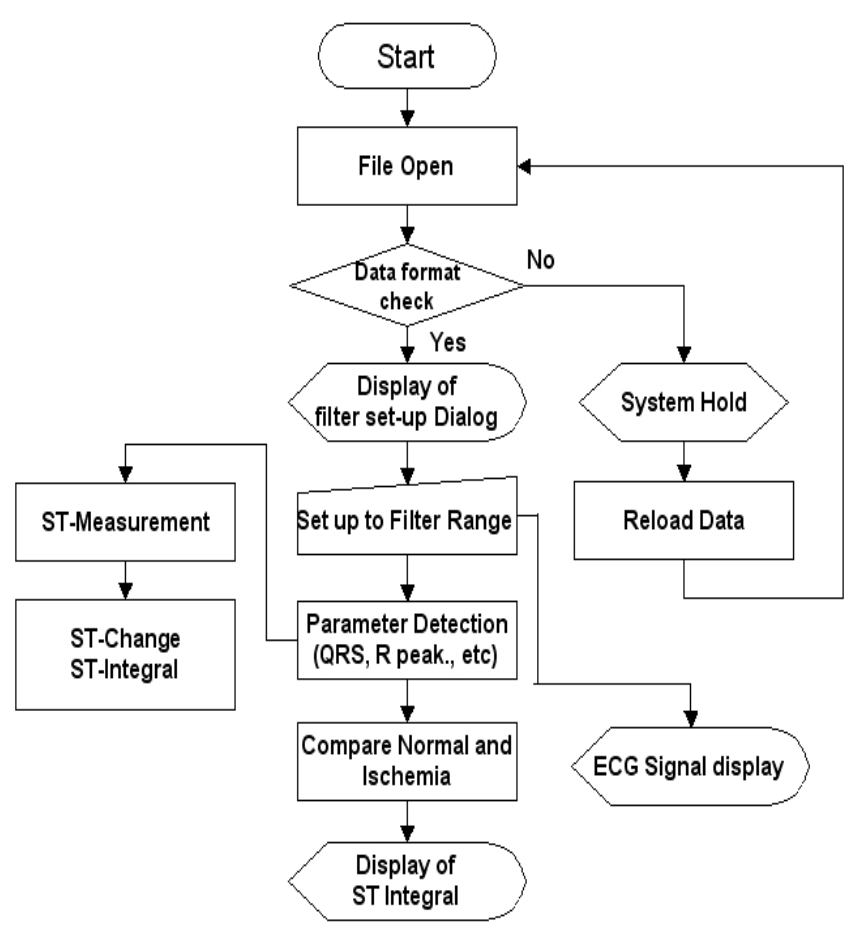

Fig. 1. Automation Detection Of ST-Segment Block Diagram

Therefore, the signal future of detection and analysis need for noise removal method. Such as, signal filtering, rhythm and beat wandering canceling. In this paper, we used FIR (Finite Impulse Response) filter method in order to remove noise, which is cancelled for low and high frequency in signal. This filtering frequency default range was set at 5 to $15 \mathrm{~Hz}$. We also used dialog option for perform to control for user's convenience and noise characteristic that it is able to filtering for variety noise band. The reduced noise signal for filter is as follows.

$$
y(n T)=\sum_{k=0}^{M} a_{k} x(n T-k T)
$$

\section{QRS complex and R-peak detection}

Many algorithms and method have been applied in QRSwave detection research. In fact, many systems have already been designed and implement to perform signal-processing tasks such as 12-lead off-line ECG analysis, Holter system analysis, and real-time patient monitoring. All these applications require an accurate detection of the QRS complex of the ECG [13]. Therefore, the precision detection of QRS complex and R-peak in the analysis of the ECG are very important and first step of signal analyze. The RR-Interval is the distance between two subsequent QRS complex and represent the Heart Rate (HR) variability. In our system, we used a robust real-time QRS detection algorithm popularly known as Pan-Tompkins algorithm [14] and added searchback compare method [7].

Pan-Tompkins algorithm has detection sensitivity for 99.3\% that it has competence for real-time ECG signal parameter detection. The algorithm includes a series of six steps: ECG signal preprocessing, derivative, squaring, integration, adaptive threshold and searchback. Figure 2 shows the block diagram for the QRS and R peak detection method.

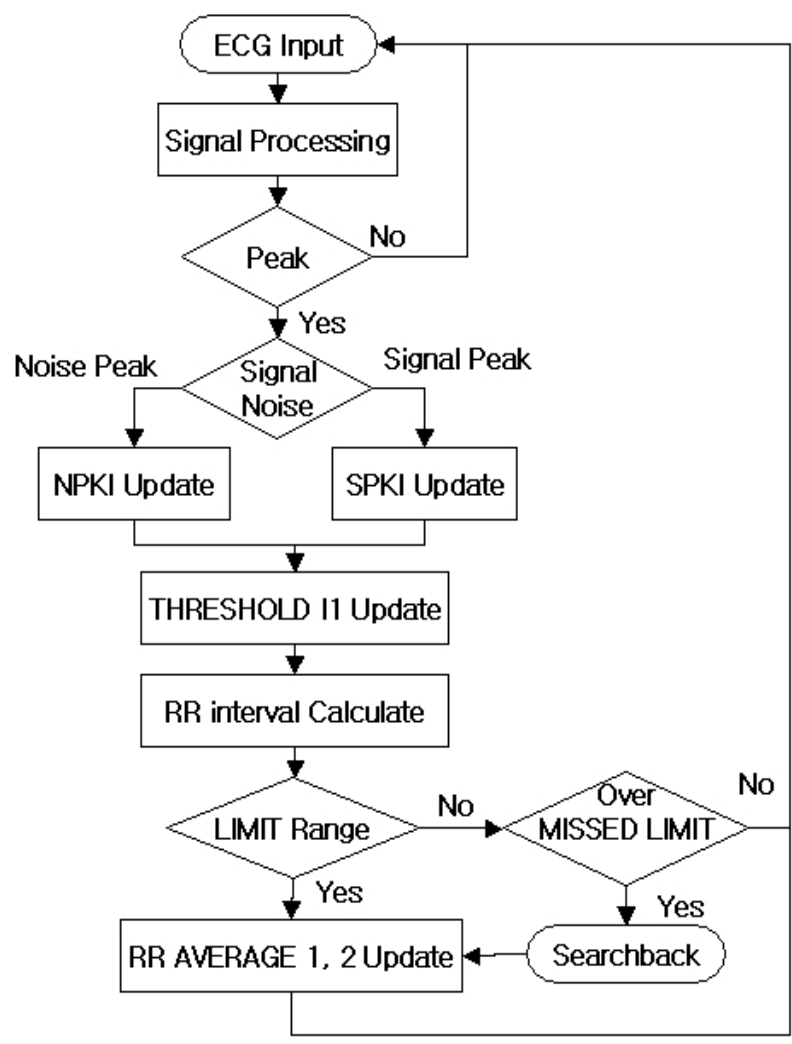

Fig. 2. QRS complex and R peak detection of block diagram

After filtering, the derivative stage provides the slop information of the QRS complex and transfer function flows:

$$
y(n)=\frac{1}{8}[2 x(n)+x(n-1)-x(n-3)-2 x(n-4)]
$$

The integration operation intensifies the slope of the frequency response curve. The output of a derivative-based operation will exhibit multiple peaks within the duration of a single QRS complex. The equation 3 is this operation.

$$
d(n)=\frac{1}{N}[x(n-(N-1))+x(n-(N-2))+\ldots+x(n)]
$$

QRS complex can be identified using general ECG parameter detection method. R-peak is easier to distinguish from noisy component since it has large amplitude [12]. So, already detected R-peak should pass verification. For deciding ultimate R-peak position by comparing to the maximum data value of a datum line in the front and rear each $10 \mathrm{msec}$ range we have verified this.

The equation 4 shows this operation and figure 3 shows the detection of the R-peaks. 
$r(n)=\frac{1}{T}[x(n)-x(n \pm 1)]$

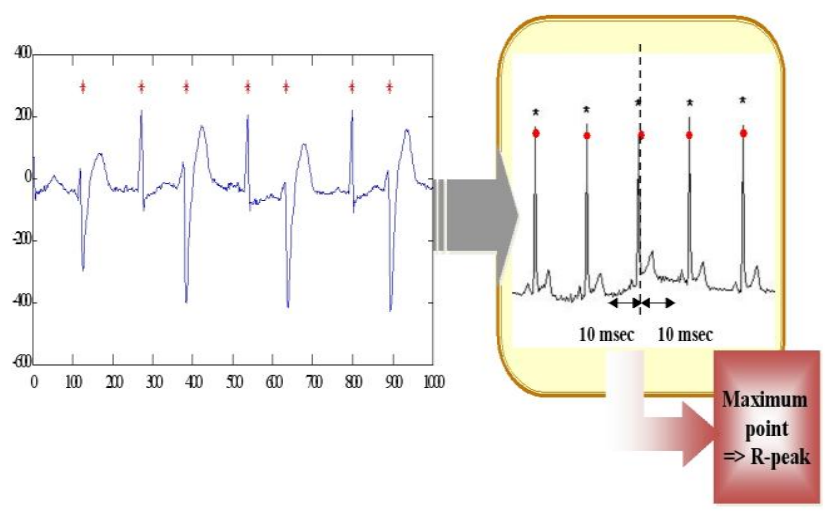

Fig. 3. Decide of the R peak detection

\section{E. Automatic detection of ST segment and measurement}

In ECG, the ST segment connects the QRS complex and the $\mathrm{T}$ wave and has duration of 0.08 to $0.12 \mathrm{sec}$ (80 to 120 msec). Abnormal ST segments are occurring by exceptional ventricular depolarization of myocardial ischemia and acute myocardial infarction. Consequently, a rising of ST's is appeared myocardial ischemia, acute endocarditis and such like things. The descent of ST's are appeared subendocardial ischemia, subendocardial infarction and such like things. That is a rising or descent of ST segment can be identified clinically that coronary artery disease. In this study, the main focus was extracting ST-segment information. Also, the preservation of the J-point was ensured. The ST-segment position is between the end of the S-wave and beginning of the T-wave [15], also the J-point where the QRS ends and the ST segment begins [16]. The calculation of the ST-segment is follows:

$$
S T_{\text {segment }}=\left(S_{\text {point }}-R_{\text {point }}\right)+140_{m \text { sec }}
$$

The three parts of ST measurement were taken from each average beats are $60 \mathrm{msec}, 80 \mathrm{msec}$ and ST-integral after the Jpoint. They are named ST60 and ST80. Figure 4 is explanation for ST measurement. A normal ECG does not showing the any ST 60 and 80 changes. Also, the calculation of the ST- integral is follows:

$$
\operatorname{Si}(n)=[\operatorname{Si}(n)+r(n)+j(n)+s(n)]-a(n)
$$

where $\boldsymbol{S i}(\boldsymbol{n})$, is the measured ST-integral from the ECG signals; $\boldsymbol{r}(\boldsymbol{n})$, the R-peak position value; $\boldsymbol{j}(\boldsymbol{n})$, the J-point position value; $\boldsymbol{s}(\boldsymbol{n})$, the ST-integral start position value; $\boldsymbol{a}(\boldsymbol{n})$, the subtraction of from R-peak position to ST segment baseline end (between $\mathrm{T}$-wave and $\mathrm{P}$-wave) position value.

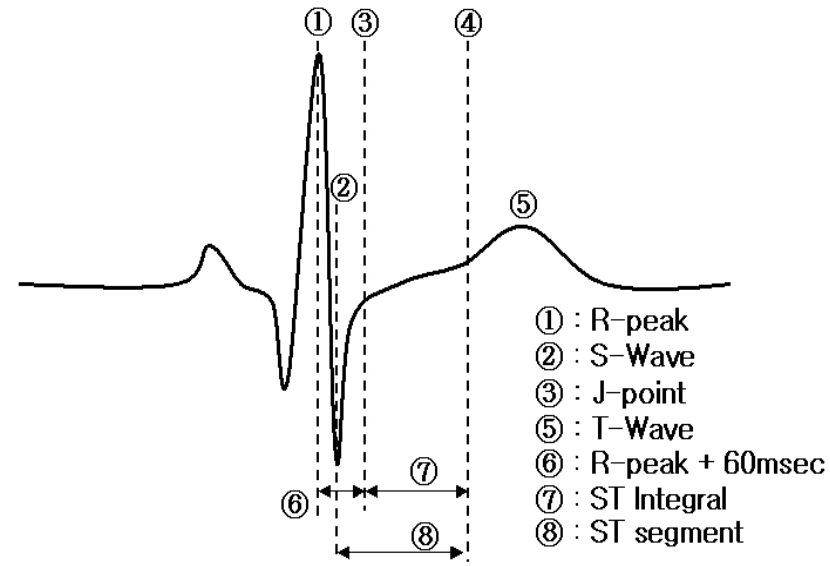

Fig. 4. ST segment and measurement

\section{EXPERIMENTS AND RESULTS}

The ECG is a cornerstone in the diagnosis of acute and chronic ischemic heart disease. The findings depend on several ischemia disease detection factors: the nature of the process versus irreversible, the duration (acute versus chronic), extent, and localization of other underlying abnormalities [17]. Hence, amplitude of ECG signal is a few $\mathrm{mV} / \mathrm{V}$ and Table 1 is explanation for waveform and characteristic normal ECG signal.

\section{TABLE I. CHARACTERISTICS OF NORMAL ECG WAVEFORM}

\begin{tabular}{|c|c||c|c|}
\hline & Amplitude (mV) & & $\begin{array}{c}\text { Parameter Duration } \\
\text { (Sec) }\end{array}$ \\
\hline \hline P Wave & 0.25 & RR Interval & $0.12-0.20$ \\
\hline R Wave & 1.60 & QRS Interval & 0.09 \\
\hline Q Wave & $25 \%$ R wave & QT Interval & $0.31-0.44$ \\
\hline T Wave & $0.1-0.5$ & ST Interval & $0.05-0.15$ \\
\hline
\end{tabular}

We tested the performance of the detection algorithm and application program used e0103 data on the European ST-T segment database. This application program has been implemented using visual $\mathrm{C}++6.0$ tools. This data was obtained from the 62 years old patient with angina and one blood vessel disease. The analysis of the application program consists of two activities. The activities are main view part and ST measurement analysis part. Figure 5 shows the application program. The main view is the original ECG signal read and operation seven-function user interface icon in Figure 5 (a); each interface icon is filter, differential, a square, windows, baseline, signal parameters position and ST measurement. The signal analysis shows (Figure 5 (b)). The set for the baseline decision of each beat which is apply to the $\mathrm{P}$ and T-wave (Figure 5 (b) in (1)); the ST parameter measurement, It is each ST-integral and ST 60/80 change (Figure 5 (b) in (2)); the ST $60 / 80$ change graph (Figure 5 (b) in (3); the ST-integral graph (Figure 5 (b) in (4)); the ST 60/HR and ST $80 / \mathrm{HR}$ graph (Figure 5 (b) in (5)). 


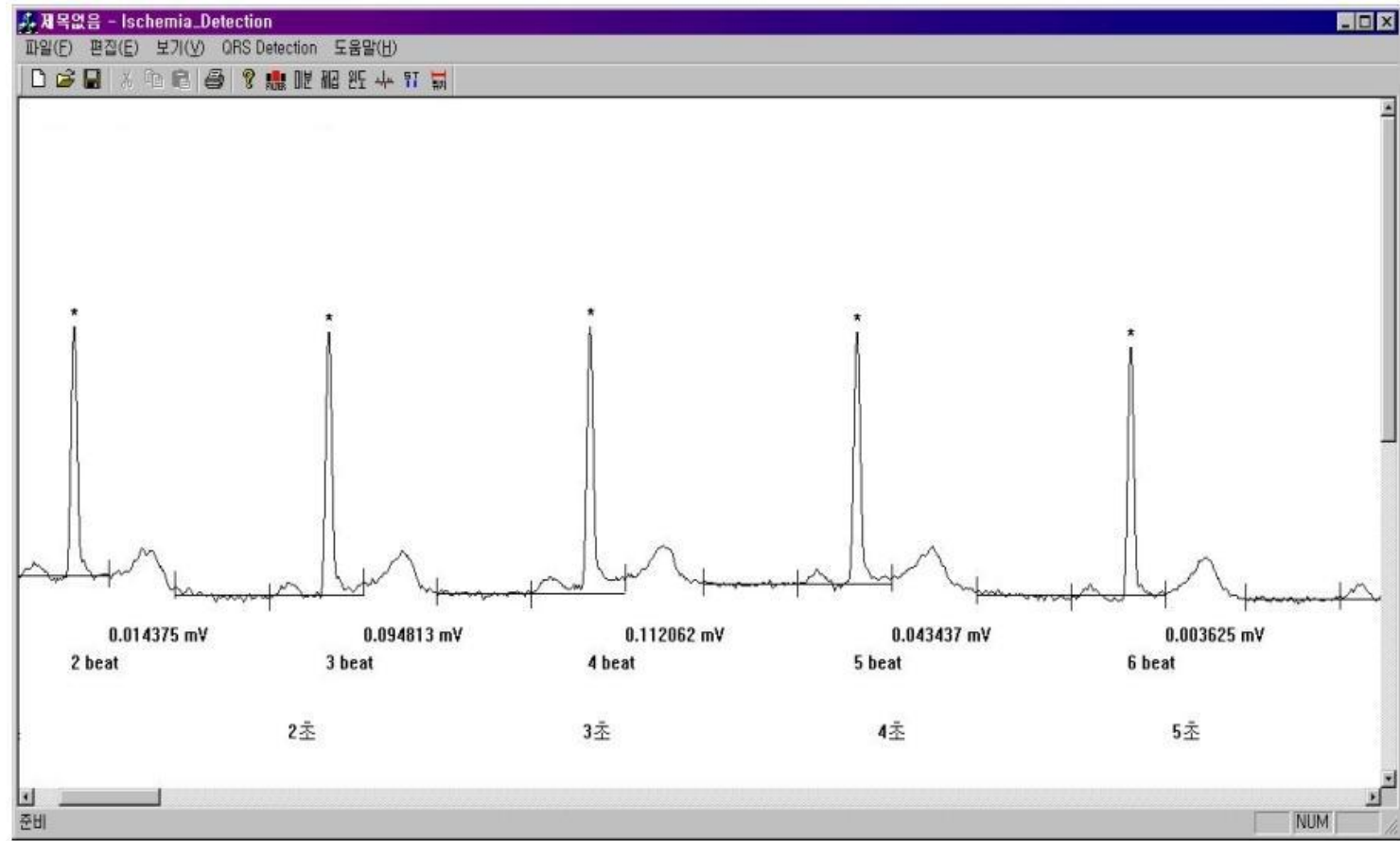

(a)

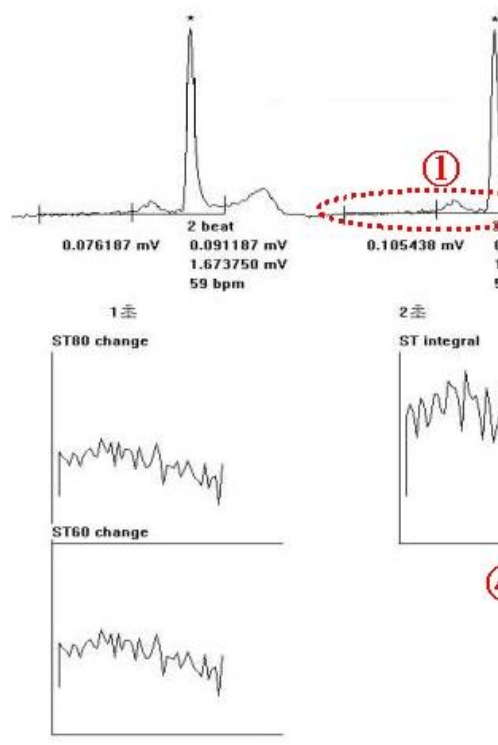

(3)

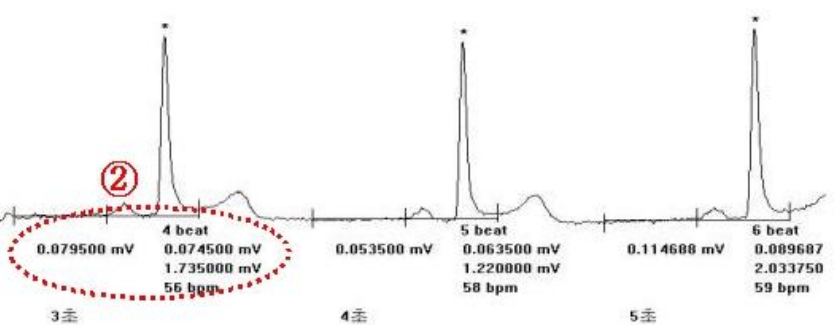

(5)

Fig. 5. Results of the ST measurement and analysis: (a) application main view; (b) signal analysis and measurement view

\section{CONCLUSIONS}

This paper presents a completely automatic algorithm and application program for component detection in ECG signal. The performance and the limitations of this method are discussed. The method and the various limits and the detection parameters used were test on just one patient's data. The performance of the method must also be assessed for the value and position of all marker positions in ECG signal. In this work, this assessment is limited to the J-point, ST60/80 and ST-segment. This signal application program experimentation is an enough successful but we have a few improvement parts and not real-time signal analysis, such as considering of the noise reduction, apply to the strong diagnostic algorithm, and totally measurement of the other physiological signals (e,g., Electromyography (EMG), Magnetoencephalography (MEG), Electroencephalography (EEG)).

Also, we would study real-time ECG signal analysis, using the algorithm for comparing the normal and abnormal ECG signals, elevation of environment design of the use interface system, testing on a long term data set, and system safety for clinical trials in a variety of conditions. Finally, the developed PC application can be useful for optimization of the already 
available diagnosis algorithms and would assist of the early heart disease detection for medical doctors.

\section{REFERENCES}

[1] T. Rocha, S. Paredes, P. Carvalho, J. Henriques, M. Harris, J. Morais, and M. Antumes, "A lead dependent ischemic episodes detection strategy using hermite functions," Biomedical Signal Processing and Control, Vol. 5, No. 4, pp.271-281, 2010.

[2] J. Garcia, L. Sornmo, S. Olmos, and P. Laguna, "Automatic detection of ST-T complex changes on the ECG using filtered RMS difference series: Application to ambulatory Ischemia monitoring," IEEE Transactions on Biomedical Engineering, Vol. 47, No. 9, pp.1195-1201, 2000.

[3] J. Garcia, P. Lander, L. L. Sornmo, S. Olmos, G. Wagner, and P. Laguna, "Comparative study of local and Karhunen-Loe've-Based ST-T indexes in recordings from human subjects with induced myocardial ischemia," Computers and Biomedical Research, vol. 31, No. 4, pp.271292, 1998.

[4] T. Barill, The Six Second ECG: A Practical Guidebook to Basic ECG Interpretation, Nursecom Educational Technologies, 2003, chapter 4.

[5] B. R. Chaitman. "The changing role of the exercise electrocardiogram as a diagnostic and prognostic test for chronic ischemic heart disease," Journal of the American College of Cardiology, Vol. 8, No. 5, pp.11951210, 1986.

[6] J. P. Martinez, S. Olmos, and P. Laguna, "T wave alternans detection: A simulation study and analysis of the European ST-T database," Computers in Cardiology, Vol. 27, pp.155-158, 2000.

[7] P. Laguna, G. B. Moody, J. Garcia, A. L. Goldberger, and R. G. Mark, "Analysis of the ST-T complex of the electrocardiogram using the Karhunen-Loe've transform: Adapative monitoring and alternans detection," Medical and Biological Engineering and Computing, Vol. 35, No. 2, pp.175-189, 1999.

[8] D. S. Rosenbaum, L. E. Jackson, J. M. Smith, H. Garan, J. N. Ruskin, and R. J. Cohen, "Electrical alternans and vulnerability to ventricular arrhythmias," The New England Journal of Medicine, Vol. 330, No. 4, pp.235-241, 1994.

[9] S. Cerutti, "In the Spotlight: Biomedical Signal Processing," IEEE Reviews in Biomedical Engineering, Vol. 1, pp.1-5, 2008.

[10] A. Taddel, G. Distante, M. Emdin, P. Pisani, G. B. Moody, C. Zeelenbere, and C. Marchesi. "The European ST-T Database: standards for evaluating systems for the analysis of ST-T changes on ambulatory electrocardigraphy," European Heart Journal, Vol. 13, No. 9, pp.1164$1172,1992$.

[11] Available: http://www.physionet.org

[12] D. H. Lee, A. Rabbi, J. Choi, and F. R. Reza. "Development of a mobile phone based e-health monitoring application," International Journal of Advanced Computer Science Applications, Vol. 3, No. 3, pp.38-43, 2012.

[13] V. X. Afonso, "ECG ARS detection," in Biomedical Digital Signal Processing, W. J. Tompkins, Ed. Englewood Cliffs, NJ: Prentice-Hall, 1993.

[14] J. Pan, and W.J. Tompkins, "A Real-Time QRS Detection Algorithm," IEEE Transactions on Biomedical Engineering, Vol. 32, No. 3, pp.230236, 1985.

[15] J. W. Hurst, "Abnormalities of the S-T segment-Part I," Clinical Cardiology, Vol. 20, pp. 511-520, 1997.

[16] J. Brownfiels, and M. Herbert, "EKG criteria for fibrinolysis: What's up with the J point?," Western Journal of Emergency Medicine, Vol. 9, No. 1, pp.40-42, 2008.

[17] A. L. Goldberger, Clinical Electrocardiography: A Simplified Approach, $7^{\text {th }}$ Edition, 2006, Chapter 11.

[18] W. D. Rosamond, L. E. Chambless et al., "Trends in the Incidence of myocardial infarction and in mortality due to coronary heart disease, 1987 to 1994," The New England Journal of Medicine, Vol. 339, No. 13, pp.861-867, 1998.

[19] D. Corrado, A. Biffi, C. Basso, A. Pelliccia, and G. Thiene, "12-lead ECG in the athlete: physiological versus pathological abnormalities," British Journal of Sports Medicine, Vol. 43, No. 9, pp.669-676, 2009.
[20] K. L. Part, K. J. Kim, and H. R. Yoon, "Application of a wavelet adaptive filter to minimize distortion of the ST-segment," Medical and Biological Engineering and Computing, Vol. 36, No. 5, pp.581-586, 1998.

[21] T. Stamkopoulos, K. Diamantaras, N. Maglaveras, and M. Strintzis, "ECG analysis using nonlinear PCA neural networks for Ischemia detection," IEEE Transactions on Signal Processing, Vol. 46, No. 11, pp.3058-3067, 1998.

[22] R. V. Andreao, B. Dorizzi, J. Boudy, and J. C. M. Mota, "ST-segment analysis using hidden markov model beat segmentation: Application to ischemia Detection," Computer in Cardiology, Vol. 31, pp.381-384, 2004.

[23] C. Papaloukas, D. I. Fotiadis, A. P. Liavas, A. Likas, and L. K. Michalis, "A knowledge based technique for automated detection of ischaemic wpisodes in long duration electrocardiograms," Medical and Biological Engineering and Computing, Vol. 39, No. 1, pp.105-112, 2001.

[24] M. Faezipour, A. Saeed, S. C. Bulusu, M. Nourani, H. Minn and L. S. Tamil, "A patient adaptive profiling scheme for ECG beat classification," IEEE Transactions on Information Technology in Biomedicine, Vol. 14, No. 5, pp.1153-1165, 2010.

[25] E. Pueyo, L. Sornmo, and P. Laguna, "ARS slops for detection and characterization of myocardial ischemia," IEEE Transactions on Biomedical Engineering, Vol. 55, No. 2, pp.468-477, 2008.

[26] Y. Birnbaum, I. Herz, S. Sclarovsky, B. Zlotikamien, A. Chetrit, L. Olmer, and G. I. Barbash, "Prognostic signficance of the admission electrocardiogram in acute myocardial infarction," Journal of the American College of Cardiology, Vol. 27, No. 5, pp.1128-1132, 1996.

Authors Profile

Duck Hee Lee received the M.Sc degree in biomedical engineering from the Hanyang University, Seoul, South Korea in 2004. From 2005 to 2009, he worked at the Biomedical Engineering Division of the National Cancer Center (NCC), South Korea, developing a surgical robot system. In 2010, he was appointed Researcher of the University of North Dakota, Biomedical Signal Processing Laboratory, USA. Since 2004, he has worked on biomedical engineering research fields. His research interests include medical device and instrument, biomedical signal processing, and surgical robotics. He authored and co-authored more ten articles journals, conference proceedings and book chapter. He is a member of Korea Society for Medical and Biological.

Jun Woo Park received the Ph.D. degree in biomedical engineering from the Seoul National University, Seoul, South Korea in 2004. Since 2004, he has worked on biomedical engineering research fields. In 2009, he was appointed Research Professor of the Department of Biomedical Engineering, College of Medicine, Korea University, Seoul, South Korea. His research interests include medical device and instrument, vision-based force feedback, telesurgery and surgical robotics. He is a member of Korea Society for Medical and Biological Engineering, Institute of Electrical and Electronics Engineers (IEEE).

Jaesoon Choi received the Ph.D. degree in biomedical engineering from the Seoul National University, Seoul, South Korea in 2003. Since 2003, he has worked on biomedical engineering research fields. From 2007 to 2012, he was appointed Research Professor of the Korea Artificial Organ Center (KAOC), Seoul, South Korea. Currently, he is Assistant Professor at Medical Engineering R\&D Center, Asan Institute for Life Science, Asan Medical Center and University of Ulsan College of Medicine, Seoul, South Korea. He was responsible for various national and international research projects focused on key components for surgery robot system. His research interests include medical device and instrument, medical fusion multi-modal simulation, VisionHaptic-Integrated Control Mechanism, and surgical robotics. He authored and coauthored more than 30 articles and holds ten patents. He is a member of Korea Society for Medical and Biological, Institute of Electrical and Electronics Engineers (IEEE), and International Society for Pediatric Mechanical Cardiopulmonary Support

Ahmed Rabbi is currently he a Ph.D. student at the Department of Electrical Engineering, University of North Dakota, USA. His research interests include biomedical signal processing and pattern recognition, heart rate monitoring using ECG, EEG movement artifacts detection and filtering, epileptic seizure detection and prediction, and human cognitive performance assessment using EEG/ECG signals, and biomedical instrumentation. He has participated as a program committee member of an international conference (ICIEV). He has published over ten articles in refereed journals, conference proceedings and 
co-authored a book chapter. He is an active member of the IEEE and IEEE Engineering in Medicine and Biology Society (EMBS).

Reza Fazel-Rezai received his BSc. and M.Sc. in Electrical Engineering and Biomedical Engineering in 1990 and 1993, respectively. He received his Ph.D. in Electrical Engineering from the University of Manitoba in Winnipeg, Canada in 1999. From 2000 to 2002, he worked in industry as a senior research scientist and research team manager. Then, he joined academia at
Sharif University of Technology and later the University of Manitoba as Assistant Professor in 2002 and 2004, respectively. Currently, he is Assistant Professor and the Director of Biomedical Signal Processing Laboratory at the Department of Electrical Engineering, University of North Dakota, USA. His research interests include biomedical engineering, signal and image processing, brain computer interface, EEG signal processing, seizure detection and prediction, neuro-feedback, and human performance evaluation based on EEG signals. 